CIENCIAMATRIA

Revista Interdisciplinaria de Humanidades, Educación, Ciencia y Tecnología

Año VI. Vol. VI. N¹1. Julio - Diciembre 2020

Hecho el depósito de ley: pp201602FA4721

ISSN-L: 2542-3029; ISSN: 2610-802X

Universidad Nacional Experimental Francisco de Miranda (UNEFM). Santa Ana de Coro. Venezuela

Adriana Marcela Manjarres-Mejia; Raysa Chiquinquirá Chirino-García

DOI 10.35381/cm.v6i11.309

\title{
Logística verde: Reto Gerencial para el manejo de la Gestión Ambiental Sostenible
}

\section{Green logistics: Management Challenge for the management of Sustainable Environmental Management}

\author{
Adriana Marcela Manjarres-Mejia \\ gestora.social.fonseca@gmail.com \\ Universidad Dr. Rafael Belloso Chacín, Maracaibo \\ Venezuela \\ https://orcid.org/0000-0002-3781-007X \\ Raysa Chiquinquirá Chirino-García \\ rchirino@urbe.edu.ve \\ Universidad Dr. Rafael Belloso Chacín, Maracaibo \\ Venezuela \\ https://orcid.org/0000-0002-0336-2870
}

Recibido: 15 de enero de 2020

Aprobado: 20 de febrero de 2020

\begin{abstract}
RESUMEN
El presente artículo, tuvo como objetivo analizar la Logística Verde como reto gerencial para el manejo de la gestión ambiental sostenible en las empresas en Colombia, para ello se utilizaron los basamentos teóricos de autores como; Chirino (2018), Ballou (2004), Gómez (2009), Dekker, Bloemhof, \& Mallidis. (2011), Embum y Zuleta (2001), Puerta, Lambraño y Cadrazco (2012), entre otros; adicionalmente, este estudio estuvo se enmarcó como investigación descriptiva documental, orientada a evaluar la importancia de la Logística verde para la Sociedad, las empresas y el Estado ante el reto empresarial dadas las operaciones antrópicas que realizan. De ahí que, el resultado de esta investigación infiere que la logística verde constituye un desafío para las empresas puesto que ésta representa para que el sector empresarial una herramienta para el manejo de la gestión ambiental sostenible donde las operaciones de las empresas en Colombia, se efectúen con criterios "verdes", desde la perspectiva del manejo de la gestión ambiental sostenible mediante el uso de tecnologías limpias y amigables con el medio ambiente para reducir las operaciones que degradan o desmejoran el medio ambiente además que amenazan la pervivencia de las generaciones futuras.
\end{abstract}




\author{
CIENCIAMATRIA \\ Revista Interdisciplinaria de Humanidades, Educación, Ciencia y Tecnología \\ Año VI. Vol. VI. N¹1. Julio - Diciembre 2020 \\ Hecho el depósito de ley: pp201602FA4721 \\ ISSN-L: 2542-3029; ISSN: 2610-802X \\ Universidad Nacional Experimental Francisco de Miranda (UNEFM). Santa Ana de Coro. Venezuela \\ Adriana Marcela Manjarres-Mejia; Raysa Chiquinquirá Chirino-García
}

Descriptores: Logística verde; gestión ambiental sostenible; reto empresarial.

\begin{abstract}
The purpose of this article was to analyze Green Logistics as a management challenge for the management of sustainable environmental management in companies in Colombia, for which the theoretical bases of authors such as; Chirino (2018), Ballou (2004), Gomez (2009), Dekker, Bloemhof, \& Mallidis. (2011), Embum and Zuleta (2001), Puerta, Lambraño and Cadrazco (2012), among others; Additionally, this study was framed as a descriptive documentary investigation, aimed at assessing the importance of Green Logistics for Society, companies and the State in the face of the business challenge given the anthropic operations they perform. Hence, the result of this research infers that green logistics is a challenge for companies since it represents for the business sector a tool for the management of sustainable environmental management where the operations of companies in Colombia are carried out. with "green" criteria, from the perspective of the management of sustainable environmental management through the use of clean and environmentally friendly technologies to reduce operations that degrade or deteriorate the environment and also threaten the survival of future generations.
\end{abstract}

Descriptors: Green logistics; sustainable environmental management; business challenge.

\title{
INTRODUCCIÓN
}

En los últimos años el fenómeno de la globalización dirigido hacia la internacionalización de las empresas ha aparejado que cada vez estas produzcan y generen cobertura en el mercado sin importar los daños que causen al ecosistema, amenaza latente que compromete la humanidad, así como la falta de toma de conciencia sobre el tema ambiental. De ahí que, hoy en día representa una prioridad en las agendas internacionales de diferentes países.

En este sentido, el tema medioambiental ha sido parte de iniciativas además de la implementación de campañas en todo el mundo; por lo que, se han realizado encuentros, conferencias, congresos, entre todo tipo de actividades. Dentro de este contexto, se ha logrado el consenso necesario para firmar acuerdos que permitan la ejercer acciones 


\section{CIENCIAMATRIA \\ Revista Interdisciplinaria de Humanidades, Educación, Ciencia y Tecnología \\ Año VI. Vol. VI. N¹1. Julio - Diciembre 2020 \\ Hecho el depósito de ley: pp201602FA4721 \\ ISSN-L: 2542-3029; ISSN: 2610-802X \\ Universidad Nacional Experimental Francisco de Miranda (UNEFM). Santa Ana de Coro. Venezuela \\ Adriana Marcela Manjarres-Mejia; Raysa Chiquinquirá Chirino-García}

para la preservación además de la conservación del ambiente, procurando minimizar los impactos, así como como los factores que lo contaminan que lo hacen vulnerables para la continuidad de los ecosistemas, las especies, incluso para la pervivencia futura de la especie humana.

Resulta perentorio, la concientización de manera general, valiéndose de los espacios académicos, así como en los empresariales, para inferir de manera general sobre logística; razón por la cual, las reflexiones teóricas que se abordan en esta investigación, se centran de modo muy particular en lo inherente a la logística verde y a la gestión ambiental sostenible, como disciplinas que buscan asociarse para la conservación del medio ambiente, así como la calidad de vida de las futuras generaciones; puesto que, coexiste una problemática originada por la falta de interés, que tanto el Estado, el sector empresarial, así como la sociedad le conceden al tema medioambiental.

Conviene resaltar que los procesos productivos aunado a las prácticas antrópicas efectuadas por las empresas a lo largo de la cadena de valor y suministros están impregnadas de impactos y riesgos, dado que estas representan una conminación implícita para el medio ambiente y el planeta. En este contexto se puede acotar que, para el sector empresarial es más relevante el aspecto económico, la rentabilidad de sus operaciones, la necesidad de optimizar el servicio al cliente sacrificando otros aspectos relevantes, como lo es la materia medioambiental. Ha llegado el momento de, inferir lo inherente a la logística desde una perspectiva general, para luego abordar de forma particular la Logística Verde.

\section{Logística}

A nivel mundial las actividades empresariales tienen un gran movimiento puesto que conlleva a la realización de innumerables exportaciones e importaciones entre diferentes países; en virtud que diariamente tiene fuerte impacto en el medio ambiente dependiendo del manejo que se le dé a cada uno de los procesos en todo el ciclo de vida de los productos en la cadena de abastecimiento. (Ballou, 2004). 


\section{CIENCIAMATRIA}

Revista Interdisciplinaria de Humanidades, Educación, Ciencia y Tecnología

Año VI. Vol. VI. N¹1. Julio - Diciembre 2020

Hecho el depósito de ley: pp201602FA4721

ISSN-L: 2542-3029; ISSN: 2610-802X

Universidad Nacional Experimental Francisco de Miranda (UNEFM). Santa Ana de Coro. Venezuela

Adriana Marcela Manjarres-Mejia; Raysa Chiquinquirá Chirino-García

Para abundar en el tema, se puede advertir que, desde una perspectiva general, la logística se concibe como un conjunto de medios y métodos ineludibles para que una organización pueda efectuar sus operaciones ya sean de servicios, distribución, almacenaje; entre otras, valiéndose para ello de la transportación de productos en el sitio justo, en el tiempo oportuno y al menor costo posible.

Por otro lado, Dekker, Bloemhof \& Mallidis (2011) sostienen que, la Logística siempre ha sido un aspecto importante dentro de las organizaciones, puesto que sirve de apoyo empresarial dada la incorporación de las nuevas tecnologías para el manejo de las operaciones, lo cual se traduce en un rol fundamental que además genera un valor agregado. Sin embargo, al elegir nuevas tecnologías no se debe obviar el "criterio verde" al decidir, pues estas deben estar en resonancia con el medio ambiente. De ahí que, en la actualidad al inferir sobre las tecnologías e información, se puede advertir que estas son significativas en un sistema logístico ya que tienen un grado de importancia análogo al de los insumos requeridos, así como al flujo financiero logístico.

Del mismo modo, es importante resaltar que la logística tiene como objetivo la satisfacción de la demanda, la cual debe ser en las mejores condiciones, gestionada mediante medios idóneos para lograrlo, movilizando de forma adecuada los recursos tanto humanos como financieros sin obviar algunos factores relevantes, tales como: los costos, calidad y seguridad del servicio y/o producto, otorgando así una ventaja competitiva y mejoramiento al margen de beneficio en la empresa.

En resumen, las investigadoras advierten que, la logística ejerce un rol estratégico en las funciones empresariales ya que estas están vinculadas a la cadena de suministros tanto de los clientes, proveedores, así como a los procesos de producción, para ulteriormente lograr la satisfacción del cliente, los cuales redundan en beneficios como lo es la reducción de costos. En tal sentido, en el próximo segmento se ilustra un diagrama que refleja la logística y la distribución 
CIENCIAMATRIA

Revista Interdisciplinaria de Humanidades, Educación, Ciencia y Tecnología

Año VI. Vol. VI. N¹1. Julio - Diciembre 2020

Hecho el depósito de ley: pp201602FA4721

ISSN-L: 2542-3029; ISSN: 2610-802X

Universidad Nacional Experimental Francisco de Miranda (UNEFM). Santa Ana de Coro. Venezuela

Adriana Marcela Manjarres-Mejia; Raysa Chiquinquirá Chirino-García

Figura No. 1

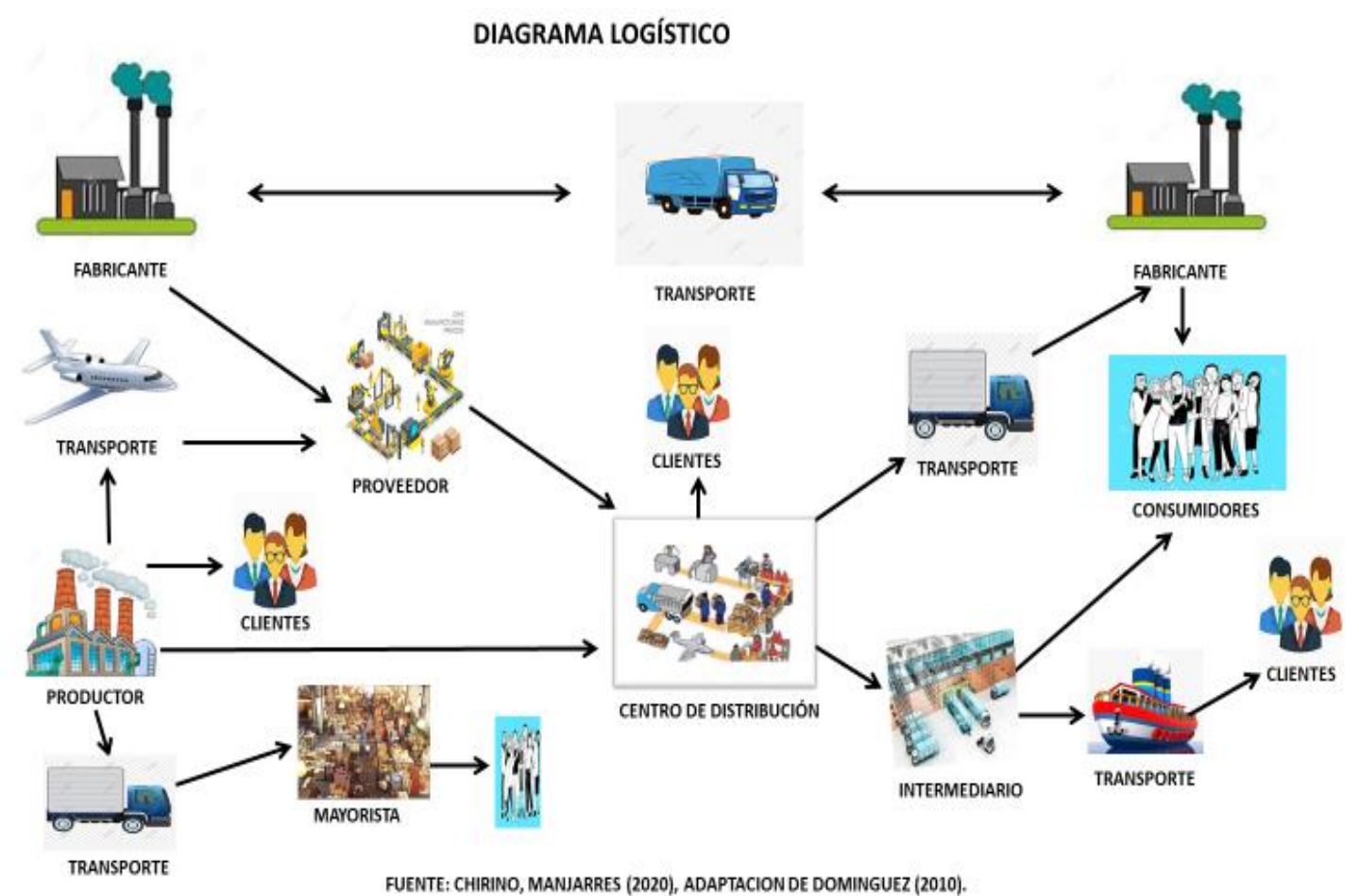

Ha llegado el momento de advertir que, Colombia ha experimentado varios fenómenos, tales como desbordamientos de ríos, pérdidas de cosechas, inviernos continuos, sequias, entre otros, tal como lo comenta Gómez (2009), Adicionalmente el diario el Tiempo (2014), divulga la muerte de hasta 20.000 chigüiros y 3.000 reses a causa de falta de agua en el departamento de Casanare aunado a la problemática resaltada se agrega el reporte de la Organización Mundial de Salud (OMS), donde en el año 2012, murieron siete millones de personas a causa de la contaminación ambiental (Disponible en: http://www.eltiempo.com/vida-de-hoy/salud/contaminacion-mato-a-sietemillones-depersonas-en-2012 13721295-421. Consultada el 22/02/2020).

Sobre la base de las ideas planteadas anteriormente, para las investigadoras el tema ambiental, así como la logística son transversales en el sector empresarial pues se 
CIENCIAMATRIA

Revista Interdisciplinaria de Humanidades, Educación, Ciencia y Tecnología

Año VI. Vol. VI. N¹1. Julio - Diciembre 2020

Hecho el depósito de ley: pp201602FA4721

ISSN-L: 2542-3029; ISSN: 2610-802X

Universidad Nacional Experimental Francisco de Miranda (UNEFM). Santa Ana de Coro. Venezuela

Adriana Marcela Manjarres-Mejia; Raysa Chiquinquirá Chirino-García

aplican diversos conceptos, tales como: logística verde, logística inversa, ecología verde, entre otras acepciones, en fin, es una iniciativa que propende analizar y reducir el impacto de desmejoramiento o degradación del medio ambiente.

\section{Logística verde}

Las problemáticas ambientales causadas por los cambios climáticos, han producido en diferentes países que se adopten medidas y celebren acuerdos, los cuales van de la mano con la preservación, así como la protección de ese gran activo que es el ambiente; por lo que, resulta perentorio un mejor uso de los recursos logísticos; ello es, emerge y se da paso a la logística verde, con la cual se puedan adecuar los procesos de manera que los mismos sean ecológicos, ahorrativos, que induzcan el desarrollo económico pero estableciendo una economía circular. En otras palabras, usando las $5 R$ s de la logística para materias primas y el aprovechamiento de desechos, almacenaje, transporte, entre otros, empero, con "criterio y mentalidad "verde.

En otro orden de ideas, Puerta, Lambraño y Cadrazco (2012) comentan que, la logística verde es entonces un modelo de economía tradicional que hace más eficiente el uso de los recursos, generando así procesos más amigables con el medio ambiente. (Disponible en:

http://45.5.172.45/bitstream/10819/1601/1/Estrategias Logistica Desarrollo

\section{Puerta 2012.pdf. Consultada el 20/02/2020).}

Por su parte, Chirino (2018) sostiene que la logística verde está referida al consumo de los recursos naturales no renovables, emisión de contaminantes, utilización de vías de acceso o comunicación, contaminación sonora y deposición de residuos, entre otros; en otras palabras, se enfoca más en la logística directa. De ahí que, es menester para una empresa que se comporte ecológicamente responsable en materia de sustentabilidad, e iniciar por integrar a su filosofía, la forma de pensar con criterio "verde" en toda la organización.

Continúa agregando Chirino (2018) que, la logística verde es una disciplina muy amplia; 
CIENCIAMATRIA

Revista Interdisciplinaria de Humanidades, Educación, Ciencia y Tecnología

Año VI. Vol. VI. N¹1. Julio - Diciembre 2020

Hecho el depósito de ley: pp201602FA4721

ISSN-L: 2542-3029; ISSN: 2610-802X

Universidad Nacional Experimental Francisco de Miranda (UNEFM). Santa Ana de Coro. Venezuela

Adriana Marcela Manjarres-Mejia; Raysa Chiquinquirá Chirino-García

por lo que, constituye un desafío para los gobiernos corporativos de las organizaciones pues su vasto beneficio contrapuesto a la logística utilizada actualmente, podrían conducir a tomar las acciones siguientes:

- Establecer en las organizaciones lucrativas una mentalidad y comportamiento "verde"

- Sobre la base del inciso anterior, implementar por parte del gobierno corporativo de la empresa medidas de control y evaluación en materia ambiental.

- Efectuar la adopción sistematizada de la logística verde; ello es, área por área para ir acogiendo el concepto establecido por gobierno corporativo además de que los todos los miembros de la organización tendrán que involucrarse y responsabilizarse con el ambiente

- Influir en la población ya sea a través de estrategias que permitan el cambio de comportamiento e ideas, sobre el consumo de productos que no afecten el ambiente.

De ahí que, las investigadoras sostienen que, la relevancia se le otorga a la logística verde, dependiendo del giro empresarial e industrial de las organizaciones, además de la realización de algunas tareas; puesto que, la alta gerencia le conceden mayor atención a los procesos relacionados con el transporte y el mercadeo para que estos se efectúen a menor costo, sin importar las emanaciones de $\mathrm{CO} 2$ y otros agentes contaminantes en la cadena de producción.

En efecto, para la gerencia de empresarial, la materia relacionada al medioambiente solo es una visión de moda ya que no genera beneficios económicos ni rentabilidad, es por ello que resulta conveniente, visualizar el proceso de logística verde con el propósito de crear conciencia para el uso eficiente energético, así como la minimización de emisiones de $\mathrm{CO} 2$, entre otras estrategias aplicables con la logística verde, tal como se ilustra en la próxima figura 
CIENCIAMATRIA

Revista Interdisciplinaria de Humanidades, Educación, Ciencia y Tecnología

Año VI. Vol. VI. N¹1. Julio - Diciembre 2020

Hecho el depósito de ley: pp201602FA4721

ISSN-L: 2542-3029; ISSN: 2610-802X

Universidad Nacional Experimental Francisco de Miranda (UNEFM). Santa Ana de Coro. Venezuela

Adriana Marcela Manjarres-Mejia; Raysa Chiquinquirá Chirino-García

Figura No. 2

PROCESO LOGÍSTICA VERDE

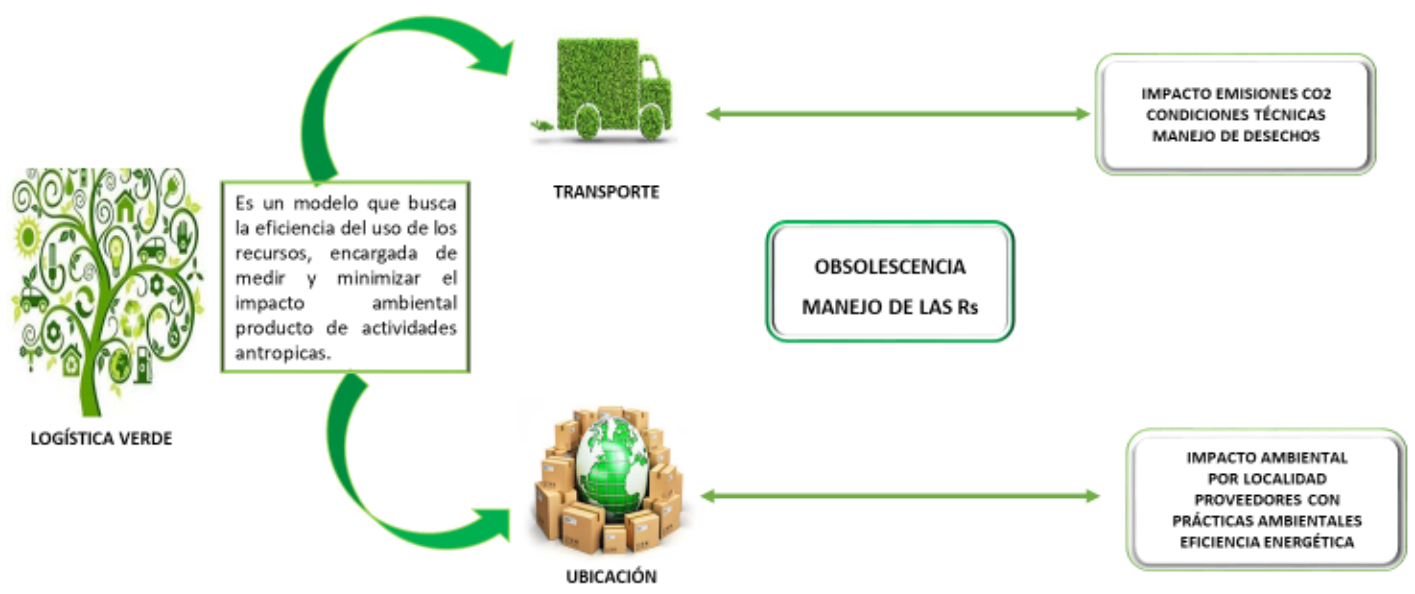

FUENTE: CHIRINO, MAJARRÉS (2020)

Ahora bien, resulta perentorio que el sector empresarial efectúe mejoras en las prácticas logísticas para la implementación del tema ambiental dado que el mal uso de energía, la utilización de materiales, así como materias primas poco amigables con el medio ambiente, dado que ello apareja una problemática que, dado el incremento de la población, así como el surgimiento de nuevas empresas e industrias agravan y amenazan las generaciones futuras.

A lo anterior se agrega, la información suministrada por Palacios (2013) quien comenta que, las fábricas a nivel mundial, los procesos de producción a nivel macro tienen gran influencia en la emisión de gases, contaminación y actividades antrópicas pues generan el $14 \%$ de las emisiones mundiales de CO2; de igual manera, la infraestructura del almacenamiento, la utilización de empaques agresivos contra el medio ambiente, entre otros.

Desde otra óptica las investigadoras advierten que, La logística verde surge como respuesta a la preocupación mundial de proteger y resguardar al medio ambiente; puesto que, el uso excesivo de recursos requiere que el sector empresarial debe propender a 
desarrollar estrategias para que sus procesos productivos se realicen con bajo un ecosistema amigable con el medio ambiente para minimizar el impacto ambiental que causa la producción de los productos a la sociedad.

De ahí que la responsabilidad social empresarial en materia ambiental debe estar orientada a reducir el impacto en el medio ambiente; por tanto, es conveniente que se revisen cada una de las fases del proceso productivo, para poder encontrar mejoras que generen beneficios medioambientales; dado que, existen empresas que al utilizar este concepto han encontrado grandes desperdicios de materias primas, materiales y recursos que no están siendo utilizados de manera adecuada además de usos innecesarios de materiales, de energía ineficiente, entre otros hallazgos que se pueden dar dentro de la cadena logística.

\section{Gestión ambiental sostenible}

La cotidianidad de la sociedad además del aumento poblacional apareja el surgimiento de conceptualizaciones relacionadas con la materia medio ambiental, puesto que los procesos que desarrolla el sector empresarial están acompañados de actividades antrópicas, las cuales causan impactos ambientales debido a la generación de residuos y/o desechos; entre otros, los cuales afectan no solo la calidad de vida de los ciudadanos sino la realidad empresarial.

En este sentido, Embum y Zuleta (2001) señalan que, se necesita una nueva actitud del hombre vinculada con el medio ambiente; de manera que se debe buscar la reducción del consumo de recursos naturales, disminuir la producción de residuos así como reutilizar y reciclar los desechos provenientes de las diferentes actividades para obtener procesos productivos limpios. Asimismo, agregan que, no existe una jerarquía en las formas de disponer los diferentes tipos de desperdicios, lo cual conlleva a que la decisión de la alta gerencia sea tomada de acuerdo con cada caso, esto induce a la concientización, así como al mejoramiento de las operaciones en las empresas. 
CIENCIAMATRIA

Revista Interdisciplinaria de Humanidades, Educación, Ciencia y Tecnología

Año VI. Vol. VI. N¹1. Julio - Diciembre 2020

Hecho el depósito de ley: pp201602FA4721

ISSN-L: 2542-3029; ISSN: 2610-802X

Universidad Nacional Experimental Francisco de Miranda (UNEFM). Santa Ana de Coro. Venezuela

Adriana Marcela Manjarres-Mejia; Raysa Chiquinquirá Chirino-García

En esta instancia, los términos sustentable y sostenible dejan entrever frases asociadas a expresiones de moda sin considerar lo que involucra ni el valor que representa para la sociedad y el mundo. Por consiguiente, para deslindar esos términos, resulta perentorio aclarar los mismos a fin de poder efectuar el abordaje de la variable Gestión Ambiental Sostenible. De ahí que, para la Cepal/Pnuma/Orpalc (2002), el término sostenible, está vinculado con el crecimiento, estabilidad social y modernización; de manera que, es necesario reconocer que es un concepto muy complejo puesto que no solo implica el factor económico o de crecimiento material, sino que también involucra la realización plena del ser humano (Disponible en: https://www.cepal.org/rio20/tpl/docs/5.DesSost.Julio2002.pdf. Consultada el 20/02/2020).

Por su parte, Espinoza (2007) comenta que el término sostenible está relacionado con la satisfacción de las necesidades del presente, sin comprometer la habilidad de las futuras generaciones para alcanzar sus propias metas. Asimismo, advierte que sustentable y sostenible son análogos, que la confusión se originó al efectuar la traducción de ambos términos en la Cumbre de la Tierra (1992) celebrada en Rio de Janeiro (Disponible en: www.un.org/spanish/esa/sustdev/agenda21/index.htm. Consultada el 20/02/2020).

No obstante, Álvarez (2015) otorga una conceptualización más moderna, pues vincula el término sostenible con el proceso de mejoramiento sostenido, así como equitativo de la calidad de vida de las personas, fundamentado en medidas apropiadas de conservación y protección ambiental, también recalcó el autor, que la sustentabilidad y el progreso son elementos que van de la mano; en otras palabras, que no puede coexistir uno sin el otro. De las definiciones expuestas anteriormente, podemos ratificar que la Sostenibilidad y Sustentabilidad no buscan frenar el progreso ni involucionar a estados primitivos ya que precisamente persiguen fomentar un progreso, pero desde un enfoque distinto y más amplio. En consecuencia, es desde ahí, donde está cimentado el verdadero reto en las empresas. 
En virtud de las conceptualizaciones planteadas anteriormente, las investigadoras observan coincidencia en las definiciones aportadas por la Cepal/Pnuma/Orpalc (2002), por la División de Desarrollo Sostenible de las Naciones Unidas "ONU" (2000) y Espinoza (2007); no obstante Álvarez (2015), concede una acepción más amplia al considerar que tanto la sostenibilidad como la sustentabilidad constituyen elementos para el progreso, los cuales van de la mano y que no puede coexistir uno sin el otro. De ahí que, se fija posición con el criterio establecido por el autor Álvarez (2015).

Volviendo la mirada a lo anterior, se puede acotar que, las empresas basan sus operaciones y centran su atención en aquellas que le proporcionen beneficios económicos o rentabilidad; por lo que, en algunos casos dependiendo del giro mercantil de estas, así como la ejecución de algunas tareas, se ubican al margen de los controles y políticas ambientales, lo cual genera un impacto en el medio ambiente, debido a la falta de implementación de una adecuada Gestión Ambiental Sostenible (GAS).

A lo anterior se agrega que, el sector empresarial al realizar la planificación tampoco considera los aspectos medio ambientales; por lo que, al omitir ese elemento relevante en la formulación de las estrategias generales de la institución, la alta gerencia se enfrentará frecuentemente a retos tras la búsqueda de técnicas cada vez más accesibles y amigables con el medio ambiente. Dentro de este contexto, la gestión ambiental sostenible está basada en el mejor uso de los recursos e impulso de un desarrollo económico mediante la creación de una economía circular.

Por tanto, resulta perentorio que las empresas, de manera particular en Colombia, ya que en el país ya se manejan criterios de "economía naranja", la cual esta centrada en la transformación de bienes y servicios; por otro lado, ella representa una oportunidad infinita para promover, fortalecer y divulgar las industrias e ideas creativas del país.

De ahí que, es recomendable exhortar al sector empresarial en el uso de materias primas amigables con el medio ambiente, almacenajes, transportes ecológicos además del procesamiento, así como el reciclaje de los desechos, entre otros. En síntesis, comenzar a aplicar las 5Rs de la Logística; puesto que, al combinarlos estratégicamente con una 


\section{CIENCIAMATRIA}

Revista Interdisciplinaria de Humanidades, Educación, Ciencia y Tecnología

Año VI. Vol. VI. N¹1. Julio - Diciembre 2020

Hecho el depósito de ley: pp201602FA4721

ISSN-L: 2542-3029; ISSN: 2610-802X

Universidad Nacional Experimental Francisco de Miranda (UNEFM). Santa Ana de Coro. Venezuela

Adriana Marcela Manjarres-Mejia; Raysa Chiquinquirá Chirino-García

difusión eficaz generará cambios de comportamientos, ideas y valores del público y empresas, así como políticas gubernamentales. Esto contribuirá con el impulso del desarrollo e implementación de una gestión ambiental sostenible.

Desde otra perspectiva, la Gestión Ambiental Sostenible en el sector empresarial, conlleva que ésta deberá estar orientada a cooperar con la conservación del medio ambiente, así como las condiciones de vida de las generaciones futuras. En este sentido, el propósito de esta investigación es generar reflexiones sobre el uso e implementación de un sistema de Gestión Ambiental Sostenible; puesto que, se evidencia poco interés por parte de gerencia empresarial sobre este delicado tema ya que no le confieren atención a esta variable, toda vez que los procesos así como las prácticas que llevan a cabo a lo largo de la cadena de valor y suministros están cargadas de actividades antrópicas que constituyen una amenaza para el desarrollo sustentable.

Dentro del mismo contexto, Chirino (2018) sostiene que la gestión ambiental sostenible, son todas aquellas diligencias conducentes al manejo integral de un sistema ambiental encauzado por acciones tendientes a lograr el desarrollo sostenible y sustentable con la aplicación de estrategias por medio de las cuales se organicen actividades para prevenir o mitigar los problemas ambientales con el fin de lograr una adecuada calidad de vida.

De ahí que, la gestión ambiental sostenible, dado que es un concepto integrador superior al del manejo ambiental, debe dar respuesta a "cómo hay que hacer" para conseguir lo planteado para lograr un desarrollo sostenible; ello es, para alcanzar un equilibrio adecuado entre el desarrollo económico, crecimiento de la población, uso racional de los recursos, protección y la conservación del ambiente, entre otros.

Ahora bien, ha llegado el momento de tomar conciencia e incorporar, al tema de la gestión ambiental sostenible algunas consideraciones, las cuales nos harán reflexionar para dar respuesta a algunas de las interrogantes que de seguidas se enuncian: ¿Por qué la gestión ambiental sostenible representa un reto para el sector empresarial en Colombia?, ¿Por qué las empresas prefieren adoptar estas prácticas o filosofías medio ambientales?, ¿Qué consecuencias pueden enfrentar esas instituciones al omitir tan delicado asunto? 
Es preciso afirmar que, existen dos ejes vinculados con el tema; por un lado, el relacionado con los esfuerzos internos que ha de desplegar la gerencia de esas empresas, en cuanto al uso eficiente energético así como, la minimización de emisión de $\mathrm{CO} 2$, para que éste no genere un impacto ambiental negativo; por otro lado, para aquellos casos que las empresas realicen exportaciones, deberán orientar sus esfuerzos para obtener la conformidad de las regulaciones ambientales extranjeras, entre otras.

Ahora bien, se debe concientizar a la población sobre este tema. En este sentido, se deben realizar esfuerzos para lograr una adecuada educación ambiental de las personas además de estimular en los empresarios el "criterio verde" de la mano de consumidores, proveedores y principalmente los directivos del sector empresarial para que asuman esa filosofía organizacional o mentalidad "verde", ya sea adaptando sus cadenas de suministro o distribución, utilizando criterios biodegradables, entre otras, puesto que si no lo adoptan, estarán al margen del desarrollo mundial pues comprometerán la calidad de vida y pervivencia de las futuras generaciones.

A lo anterior se añade que, el Estado debe contribuir con el establecimiento y divulgación de verdaderas políticas medioambientales para el manejo de desperdicios y desechos, lo cual coadyuva en la recolección, clasificación y diferenciación de los residuos y/o desechos, no solo domiciliarios sino comerciales e industriales; asimismo, estimular el reciclado mecánico o térmico y el compostaje de las fracciones orgánicas, entre otras acciones.

Desde otra perspectiva, dentro de los retos a los que se enfrenta la gerencia del sector empresarial, al efectuar la implementación de la gestión ambiental sostenible, está en que, éstas deben tomar en consideración los factores ambientales que tengan mayor influencia en el lugar donde operan los procesos productivos, por cuanto existen elementos que definirán sobre qué actividades se va a trabajar para minimizar el impacto ecológico pero ello no obsta que, la aplicación de esta filosofía sea adoptada a nivel global. 
CIENCIAMATRIA

Revista Interdisciplinaria de Humanidades, Educación, Ciencia y Tecnología

Año VI. Vol. VI. N¹1. Julio - Diciembre 2020

Hecho el depósito de ley: pp201602FA4721

ISSN-L: 2542-3029; ISSN: 2610-802X

Universidad Nacional Experimental Francisco de Miranda (UNEFM). Santa Ana de Coro. Venezuela

Adriana Marcela Manjarres-Mejia; Raysa Chiquinquirá Chirino-García

Aunado a lo anterior, la gerencia debe procurar la combinación del comportamiento del público de la mano con el Estado para impulsar no solo el desarrollo de la economía sino concentrándose en los elementos fundamentales que permitan atomizar las amenazas al medio ambiente, tales como: realizar con regularidad una evaluación del impacto ambiental (EIA) puesto, que mediante este contribuye a la detección temprana del impacto ambiental para que facilite la toma de decisiones oportuna, incorporar los costos de las medidas de protección ambiental. En efecto, aporta alternativas creativas y eficientes. Del mismo modo, compatibiliza las acciones de mejoras en las políticas, entre otros.

En síntesis, las investigadoras consideran que, la clave para lograr una gestión ambiental sostenible radica en la implementación de estrategias que busquen a maximizar la eficiencia en el manejo de los recursos. Sostienen que, se debe propender a conseguir y aplicar nuevas fuentes de materia prima, sin obviar las que se tienen, puesto que es importante, para lograr mantener los costos dentro de los rangos preestablecidos además que, representa una fuente de potenciales beneficios.

Por otro lado, infieren que, a pesar de la idea que hay que conservar las cantidades y calidades necesarias, en este escenario no se puede omitir que existirá un aumento del deterioro ambiental; si, por ejemplo, la empresa labora con materiales cuyos componentes fundamentales sean los árboles, habrá que talar mayor cantidad de ellos para poder trabajar, lo cual implicaría degradación del medio ambiente. En otras palabras, las empresas deben perseguir una gestión ambiental sostenible, para que se efectúe un consumo consciente, balaceado y coherente de los recursos naturales con el fin de proteger no solo la calidad y cantidad de este sino para garantizarle a las generaciones futuras que también podrán disponer de un medio ambiente limpio y sano. 


\section{METODOLOGÍA APLICADA}

Esta investigación se encuentra definida por el contenido que se aborda. En este sentido, el tipo de investigación es documental. En este sentido, Hernández (2016) consideran la investigación documental como aquel "estudio sistemático, secuencial, formal y teórico que recolecta, científicamente los datos. Debido a ello, este tipo de investigación se estructura por la complementariedad seleccionada, clasifica, evalúa y efectúa el análisis del contenido de material ya sea empírico, impreso o mediante medios electrónicos, entre otros, que servirán de fuente para una investigación, de un estudio documental bibliográfico, donde se analizó desde una perspectiva determinista al asumir un cuadro de categorías que permitió orientar el presente estudio.

\section{REFLEXIONES FINALES}

En síntesis, las investigadoras sostienen que la logística verde constituye un reto gerencial para el manejo de la gestión ambiental sostenible en las empresas en Colombia, puesto que la clave para la alta gerencia es que deben realizar esfuerzos para lograr la eficiencia en el manejo de recursos, no solo por motivos medio ambientales, sino para poder negociar de forma eficiente los productos reintroducidos por diferentes motivos en la cadena de suministro; puesto que existen muy pocas empresas que utilizan la logística verde, así como la gestión ambiental sostenible como estrategias para mantener de forma adecuada los procesos de almacenaje, suministro, distribución, reciclado y gestión de residuos, sustitución de materiales, entre otras.

A lo anterior se agrega que, el sector empresarial deben aplicar estrategias para lograr mantener así como preservar el ambiente, puesto que la gestión ambiental sostenible se caracteriza fundamentalmente por la minimización del impacto ambiental negativo además que esto puede determinar disparidades notables sobre todo al calcular el costobeneficio; el tipo de producto o material que se recogerá debido a los obstáculos que supondrían algunos materiales así como a la necesidad de recuperarlos aunado a la actividad que se hará con este una vez retornado a la fábrica. 
CIENCIAMATRIA

Revista Interdisciplinaria de Humanidades, Educación, Ciencia y Tecnología

Año VI. Vol. VI. N¹1. Julio - Diciembre 2020

Hecho el depósito de ley: pp201602FA4721

ISSN-L: 2542-3029; ISSN: 2610-802X

Universidad Nacional Experimental Francisco de Miranda (UNEFM). Santa Ana de Coro. Venezuela

Adriana Marcela Manjarres-Mejia; Raysa Chiquinquirá Chirino-García

En otro orden de ideas, en los países del tercer mundo, la ausencia de una cultura ambiental complica la implementación de estrategias de protección medio ambiental así como la aplicación de la gestión ambiental sostenible, que al agregar la falta de interés del Estado por inexistencias de políticas en la materia, control y seguimiento aunado al incumplimiento de la normativa legal medio ambiental, pues se habla más de lo que finalmente se aplica o se norma al respecto, apenas alcanzamos a ver algunos esfuerzos tímidos realizados por algunas organizaciones, lo cual no representa un avance en la materia.

En esta instancia, las investigadoras sostienen que para implementar una gestión ambiental sostenible se debe producir una mentalización, así como cooperación total entre todos los actores intervinientes en el proceso, tales como: proveedores, capital humano, distribución, transporte, y usuario final pues cada día es mayor la demanda de estos en los distintos países. Del mismo modo, sostienen que la forma adecuada de implementar y utilizar una gestión ambiental sostenible, puede representar factores de cambio, así como generador de oportunidades que permitan romper con el paradigma; puesto que este término no solo está asociado a una moda efímera ni a la generación de costos adicionales a las empresas.

Finalmente, las investigadoras ratifican que logística verde constituye un reto gerencial para el manejo de la gestión ambiental sostenible en las empresas en Colombia ya que su implementación y utilización demandan la adopción de factores de cambio, al tiempo que representan un nicho de oportunidades empresariales que coadyuvara con la ruptura del paradigma sobre las variables estudiadas, pues en la mayoría de los casos están asociados a una moda efímera que generaría costos adicionales a las empresas. 
CIENCIAMATRIA

Revista Interdisciplinaria de Humanidades, Educación, Ciencia y Tecnología

Año VI. Vol. VI. N¹1. Julio - Diciembre 2020

Hecho el depósito de ley: pp201602FA4721

ISSN-L: 2542-3029; ISSN: 2610-802X

Universidad Nacional Experimental Francisco de Miranda (UNEFM). Santa Ana de Coro. Venezuela

Adriana Marcela Manjarres-Mejia; Raysa Chiquinquirá Chirino-García

\section{REFERENCIAS CONSULTADAS}

1. Álvarez, C (2005). Implantación de un Sistema de Gestión Corporativo. Albacete. España. (Disponible

en: http//www.mds.es/medioambiente/archivo/memoriaMA.pdf. Consultado el 20/02/2020).

2. Ballou, R. (2004). Logística ración de la cadena de suministro. (Disponible en: http://books.google.es/books?hl=es\&lr=\&id=ii5xqLQ5VLgC\&oi=fnd\&pg=PA1\&dq= la+cadena+de+abastecimiento+y+el+medio+ambiente\&ots=u17DrpHm6f\&sig =dH iRJZ2YachzJ01S7sGkN. Consultada el 20/02/2020).

3. Chirino, R (2015). Mercadeo Social como estrategia de promoción en la Gestión Ambiental. Universidad Privada Dr. Rafael Belloso Chacín. Tesis Doctoral, Mención: Publicación. (2015).

4. Chirino, R (2018). Logística verde y gestión ambiental: Desafío para el gobierno corporativo en las organizaciones lucrativas. (Disponible en: https://ijrdo.org/index.php/sshr/article/view/1966. Consultado el 22 de Febrero de 2020).

5. Dekker, R., Bloemhof, J., \& Mallidis, I. (2011). Operations Research for green logistics - Anover view of aspects, issues, contributions and challenges. European Journal of Operational Research, $671-679$. Europa.

6. El tiempo 25 de marzo de 2014 Siete millones de muertes en 2012 son culpa de la contaminación: (Disponible en: http://www.eltiempo.com/vida-dehoy/salud/contaminacion-mato-a-sietemillones-de-personas-en2012 13721295-421. Consultada el 22/02/2020).

7. Embum, X., Zuleta, A. (2001). Sistemas de Gestión Medioambiental. Editorial Colex, Madrid-España.

8. Espinoza, G (2007). Gestión y fundamentos de evaluación de impacto ambiental. Banco interamicano de desarrollo (BID). Santiago de Chile. Centro de estudios para el desarrollo-CED.

9. Gómez, J (2009). Vulnerabilidad y medio ambiente. (Disponible en: https://studylib.es/doc/5647448/vulnerabilidad-y-medioambiente-jos\%C3\%A9-javier-g\%C3\%B3mez. Consultada el 22/02/2020). 


\section{CIENCIAMATRIA}

Revista Interdisciplinaria de Humanidades, Educación, Ciencia y Tecnología

Año VI. Vol. VI. N¹1. Julio - Diciembre 2020

Hecho el depósito de ley: pp201602FA4721

ISSN-L: 2542-3029; ISSN: 2610-802X

Universidad Nacional Experimental Francisco de Miranda (UNEFM). Santa Ana de Coro. Venezuela

Adriana Marcela Manjarres-Mejia; Raysa Chiquinquirá Chirino-García

10. Hernández y otros (2016). Metodología de la investigación. Sexta edición. Editorial Mc Graw Hill. México. D.F.

11.Palacios, A (2013). Legiscomex.com. Entrevista del 21 de Agosto, al encargado del programa de logística verde Gogreen para América de la empresa de transporte de carga DHL Global Forwarding, (Disponible en: http://www.legiscomex.com/BancoConocimiento/L/logisticaverde-actualizacion-dhl/logisticaverde-actualizacion-dhl.asp. Consultada el 20/02/2020).

12. Puerta, Lambraño y Cadrazco (2012). Estrategias logísticas para un desarrollo sostenible. Universidad San Buenaventura. Medellín. Colombia.

(C2020 por los autores. Este artículo es de acceso abierto y distribuido según los términos y condiciones de la licencia Creative Commons Atribución-NoComercial-Compartirlgual 4.0 Internacional (CC BY-NC-SA 4.0) (https://creativecommons.org/licenses/by-nc-sa/4.0/). 